Mon. Not. R. Astron. Soc. 000, 000-000 (0000) Printed 28 October $2019 \quad$ (MN LATEX style file v2.2)

\title{
From diffuse extragalactic and galactic gamma rays to limits on extra dimensions
}

\author{
Michel Cassé ${ }^{1}$, Bruno Mansoulié ${ }^{1}$, Joseph Silk Si, $^{2,4}$ \\ ${ }^{1}$ IRFU, CEA, University Paris-Saclay, Gif-sur-Yvette, France \\ 2 Institut d'Astrophysique de Paris, CNRS, 98bis bd Arago, 75014 Paris, France \\ 3 Dept of Physics \& Astronomy, The Johns Hopkins University, Baltimore MD 21218, USA \\ ${ }^{4}$ Beecroft Institute of Particle Astrophysics and Cosmology, Department of Physics, University of Oxford, 1 Keble Road Oxford, OX1 3RH, UK
}

28 October 2019

\begin{abstract}
We derive the maximum fraction of energy emitted in the form of massive (KaluzaKlein) gravitons by core collapse supernovae, and the corresponding minimal extradimensional Planck mass $\mathrm{M}^{*}$ in the ADD gravity framework at TeV-scales. Our constraints arise: a) from the extragalactic gamma ray background observed by FermiLAT after astrophysical sources have been removed, and b) via the residual galactic emission left after astrophysical and potentially dark matter emission have been removed. We focus on a number of extra dimensions 3 and 4 , since $\mathrm{M}^{*}$ is then in the $\mathrm{TeV}$ range, where astrophysical and collider constraints compete. Lower limits on $\mathrm{M}^{*}$ are derived in case a) of $8.0 \mathrm{TeV}$ and $1.1 \mathrm{TeV}$, and in case b) of $16 \mathrm{TeV}$ and $1.9 \mathrm{TeV}$, for a number of extra dimensions $n=3$ and $n=4$ respectively. These limits are especially robust and insensitive to the various uncertainties involved.
\end{abstract}

Key words: gravitation- gamma-rays: diffuse background

\section{INTRODUCTION}

The standard model of particle physics is compelling but it fails to explain why gravity is so much weaker than the ' electromagnetic or nuclear forces. The huge difference in strength of fundamental forces is one aspect of the "hier" archy problem"; another is the Higgs boson mass which is much lower than it should be in the absence of supersymmetry. This is manifested by the difference between the ' electroweak and gravitational energy scales (about $1 \mathrm{TeV}$ against $10^{16} \mathrm{TeV}$ ) and the difficulty of its stabilisation (Buchmueller et al. 2012). Hidden extra dimensions have been invoked to dilute gravity and make it appear feeble (Arkani-Hamed et al.1999, Randall and Sundrum 1999) whereas in the extradimensional realm, gravity is as strong as electromagnetism.

In the following, we adopt the ADD framework (Arkani-Hamed et al.1999) expected to give a purely geometric explanation to the hierarchy puzzles (why is $\mathrm{G}$ so feeble? why is the Planck mass so high?). In this framework, inspired by the Kaluza-Klein hypothesis of the existence of compact extra dimensions (ED), quantum gravity features providentially at about the TeV energy scale, which renders it accessible to experimental and observational verification through the transient existence of massive, unstable, Kaluza-Klein gravitons (hereafter referred to as gravitons). This bold hypothesis takes the risk of being tested physically in the laboratory (table-top experiments, via spectroscopy at CERN, and astrophysically through gamma ray astronomy. Cosmological constraints are stringent (Hannestad and Raffelt 2001) but more uncertain than those discussed here. We derive robust limits on the fundamental (extra-dimensional) Planck mass $\mathrm{M}^{*}$ for a number of $\mathrm{ED}, n=3$ and 4 , from gamma ray observations, and compare these results with those derived from LHC experiments. For $\mathrm{M}^{*}$, we adopt the convention of (Hannestad and Raffelt 2003), defining $\mathrm{M}^{*}$ through the formula $M_{P l} /(8 \pi)=(2 \pi R)^{n}\left(M^{*}\right)^{n+2}$.

Note that ED do not "explain" the weakness of gravitation but geometrize the puzzle. Thus, if we assume that large ED exist in nature, they would inevitably have been present in the past, but today are compactified. However at high energies, there is a potential signature via the generation of gravitons which would be emitted in supernova (SN) cores with energies around $100 \mathrm{MeV}$, providing a significant contribution to the celestial diffuse gamma ray background via radiative decays. This potential contribution allows us to set independent limits from those deduced from individual neutron stars, around which gravitons are trapped and decay into gamma rays (Hannestad and Raffelt 2001. Hannestad and Raffelt 2002), most recently constrained by Fermi data (Fermi-LAT Collaboration et al. 2012) as 
well as by neutron stars grouped in the galactic bulge (Cassé et al. 2004). High energy neutrino bounds have also been examined but are generally weaker (Astashenkov and Kisselev 2018). In this Letter, we derive the maximum fraction of energy emitted in the form of gravitons by all core collapse SN, failed or not, and assess the corresponding minimal extra-dimensional Planck mass. Our limits are constrained by both the extragalactic isotropic gamma ray background and the diffuse galactic emission, less galactic centre excess associated either with dark matter annihilations or millisecond pulsars observed by the Fermi LAT after astrophysical sources have been removed. We focus on a number of ED 3 and 4, since the fundamental Planck mass is then in the $\mathrm{TeV}$ range, where astrophysical and collider constraints compete. Its value is robust and insensitive to the various uncertainties involved.

\section{EXTRA DIMENSIONS AND THE ISOTROPIC GAMMA RAY BACKGROUND}

We focus on the isotropic extragalactic gamma ray background produced by all SN from $\mathrm{z}=0$ to $z \sim 5$, updating the seminal earlier work (Hannestad and Raffelt 2001) and assuming, like the authors, a toroidal compactification of $\mathrm{ED}$, all with the same radius. In general, during core collapse, matter reaches such a high average temperature $(\sim$ $30 \mathrm{MeV}$ ) that gravitons could be abundantly produced. Due to the extreme weakness of the gravitational force, they escape the dense stellar core, unimpeded. Each graviton of mass $m$ subsequently decays in space into two gamma rays, of $\mathrm{m} / 2$ energy, producing a diffuse gamma ray background that could be observable for large enough ED or sufficiently small extra-dimensional Planck mass $\mathrm{M}^{*}$. Since this is not the case, we derive a lower bound on $\mathrm{M}^{*}$.

The value of $\mathrm{M}^{*}$ is proportional to $f_{K K}^{-1 /(n+2)}$ (Hannestad and Raffelt 2002), where $f_{K K}$ is the fraction of energy emitted in the form of Kaluza-Klein gravitons during the core-collapse of a SN. Hence its value is especially robust and insensitive to the various uncertainties involved in this method, most notably mean temperature during core collapse, equation of state of superdense matter, stellar evolutionary model, explosion simulation, SN rate, model of galactic evolution, and fraction of the gamma ray background left in photons from graviton decays.

We take advantage of detailed calculations of the extragalactic antineutrino flux arising from SN (successful or failed) based on hydrodynamical simulations of the Garching group (MØller et al. 2018, Priya and Lunardini 2017) (and references therein) along with reasonable models of galactic evolution to derive the hypothetical Isotropic Gamma Ray Background (GRB) arising from graviton decay and compare it to the residuals left by astrophysical sources. Indeed, the estimate of (Hannestad and Raffelt 2001) based on the GRB produced by all SN since the creation of the first stars is excessively conservative (no evolution of the $\mathrm{SN}$ rate as a function of redshift, no failed SN, all the observed emission not surpassed by graviton decay). It is better to use the residuals left after all the contributions of astrophysical sources have been removed, which is good practice in searches for dark matter signatures. Indeed, little room is left for exotic processes (dark matter, primordial black holes, quasars and blazars) since the GRB is well explained by the gamma ray emission from galaxies, quasars and blazars (Ackermann et al. 2016)

The energy of a typical SN is $3 \times 10^{53}$ ergs or $1.8 \times$ $10^{59} \mathrm{MeV}$ and the number of electron antineutrinos released (with a mean energy of $\sim 15 \mathrm{MeV}$ ) is $2 \times 10^{57}$. If all the energy is emitted in the form of gravitons $\left(f_{K K}\right.$ $=1$ ) with an energy of about $100 \mathrm{MeV}$ (see Table 2 in (Hannestad and Raffelt 2003) ), the number of gravitons emitted is $15 \%$ that of antineutrinos, and the corresponding gamma ray emission is $30 \%$, since each graviton decays into two gammas. Empirically, there is little room left to nonastrophysical sources (dark matter annihilation or decay and/or graviton decay.) The maximum gamma ray flux left for exotic sources above $100 \mathrm{MeV}$ is $\sim 6 \times 10^{-7} \mathrm{~cm}^{-2} \mathrm{~s}^{-1} \mathrm{sr}^{-1}$ (Fermi-LAT Collaboration et al. 2012), which we safely extrapolate to a flux above $50 \mathrm{MeV}$ of $3 \times 10^{-6} \mathrm{~cm}^{-2} \mathrm{~s}^{-1} \mathrm{sr}^{-1}$ or $4 \times 10^{-5} \mathrm{~cm}^{-2} \mathrm{~s}^{-1}$, against $\sim 1 \mathrm{~cm}^{-2} \mathrm{~s}^{-1}$ deduced from the calculated (still unobserved) antineutrino background (Priya and Lunardini 2017). So $f_{K K}$ is about $4 \times 10^{-5}$. The exact value is not of crucial importance since $\mathrm{M}^{*}$ is proportional to $f_{K K}^{1 / 5}$ or $f_{K K}^{1 / 6}$ for $\mathrm{n}=3$ and 4 , thus order of magnitude estimates are sufficient.

Consequently, $f_{K K}$ is $\sim 250$ times lower than that assumed in (Hannestad and Raffelt 2003. Hannestad and Raffelt 2004) $\left(10^{-2}\right)$. Using values from Table VI in the same reference, we derive the limits $\mathrm{M}^{*}>$ 8.0 TeV and $1.1 \mathrm{TeV}$ for $\mathrm{n}=3$ and 4 . These limits can be compared to those obtained at the LHC by the ATLAS and CMS collaborations searching for direct production of gravitons together with one or several jets, namely ATLAS: $2.1 \mathrm{TeV}$ and 1.6 TeV (ATLAS collaboration 2018) and CMS: 2.5 and $1.9 \mathrm{TeV}$ (CMS collaboration 2018-1) for $n=3$ and $n=4$ respectively, or by CMS searching for a contribution to dijet production through virtual graviton exchange: 4.0 TeV and 3.0 TeV (CMS collaboration 2018-2) for $n=3$ and $n=4$ respectively. The constraints from this analysis are found to be of the same order of magnitude as those from the LHC, more stringent in the case $n=3$ and less so in the case $n=4$. Indeed the limits derived from the GRB are very robust. A factor of 10 uncertainty on $f_{K K}$ translates only into a factor 1.4 uncertainty on $\mathrm{M}^{*}$ (min) for $\mathrm{n}=4$. Thus this method liberates us from the tyranny of precision.

\subsection{Approximations}

Concerning the energy range, the gamma ray emission of gravitons is limited to about $300 \mathrm{MeV}$ whereas we have considered the whole photon spectrum for simplicity. This makes little difference due to the steepness of the GRB (slope of -2.3).

With regard to undecayed gravitons, the lifetime of gravitons is very long, about $(100 \mathrm{MeV} / \mathrm{m})^{3}$ Gyr, with $\mathrm{m}$ being their mean mass (68 and $95 \mathrm{MeV}$ for $\mathrm{n}=3$ and 4 ((Hannestad and Raffelt 2003), Table 2). Their lifetime is of the order of 1 Gyr for $\mathrm{n}=3$ and 4 , corresponding to a redshift about 0.1 . From the calculated extragalactic antineutrino background which peaks around $5 \mathrm{MeV}$ (MØller et al. 2018, Priya and Lunardini 2017) whereas the mean emission energy of SN antineutrinos is about 15 $\mathrm{MeV}$ (weighted average over the Initial Mass Function 
(Horiuchi et al. 2018), we deduce that the maximum contribution of SN to the background is at $z \sim 2$, corresponding to the maximum in the cosmic star formation rate.

\subsection{Uncertainties}

There are many sources of uncertainties, the principal one being that of the calculated neutrino flux, in turn due to that of the present core collapse SN rate (MØller et al. 2018 Priya and Lunardini 2017), by a factor of the order of 2 .

The uncertainty in the mean temperature during core collapse is much less dramatic than in the case of individual neutron stars (Hannestad and Raffelt 2003 used to constrain $\mathrm{M}^{*}$. Here $\mathrm{T}=30 \mathrm{MeV}$ is the minimum value of the mean temperature during core collapse. The bounds are quite insensitive to the assumed temperature of the emitting medium because, for a larger temperature, the average energy of the emitted KK states increases, leading to a decrease in their total number, and at the same time, the energy of the decay photons is distributed over a broader range of energies, further decreasing the differential flux. Therefore, the predicted photon flux is lower, but extends to larger energies. On the other hand, the measured gamma ray flux falls approximately as $E_{\gamma}^{-2}$ canceling the previous effect. Finally, note that the bounds derived in (Cassé et al. 2004), based on the gamma ray emission of the (old) galactic bulge, especially for $n=3$ should be reassessed since most of the gravitons would have decayed, lessening the flux from the bulge. On the other hand, one should also consider not the whole gamma ray emission of the bulge but the residual emission left by photons from graviton decays, which is difficult to ascertain. Thus the present limit on $\mathrm{n}=3$ replaces the previous one. Note once again that constraints on ED from the observations of gravitational waves (Pardo et al. 2018), indicating that gravitational and electromagnetic waves propagate at the same velocity, do not apply in our case since ED are compact (their radius is much less than the wavelengths of gravitational waves).

\section{GALACTIC GAMMA RAYS AND EXTRA DIMENSIONS}

Assuming the ADD scenario, we calculate the gamma ray luminosity of the Milky Way in erg s ${ }^{-1}$, induced by the decays of gravitons produced by core collapse and trapped around compact objects, neutrons stars and black holes (remnants of successful and failed SN). We then compare this to the space left by gamma rays produced by cosmic rays and young pulsars i.e. the residuals (calculated and observed) of the galactic emission above $100 \mathrm{MeV}$.1

The influential parameters are:

- $r_{S N}$, the SN birth rate and $r_{B H}$ the black hole(BH) birth rate, taken as 2 /century and 0.15 /century, respectively

- $M_{B H}$, the mean mass of stellar $\mathrm{BH} \sim 10 \mathrm{M}_{\odot}$

- $E_{\text {grav }}$, the total (gravitational) energy released in core collapse, $\left(\sim M^{2} / R, 3 \times 10^{53}\right.$ and $4.5 \times 10^{54} \mathrm{erg}$, respectively;

1 Millisecond pulsars are too old to contribute, all gravitons should have decayed.

\begin{tabular}{cccccc}
\hline \hline & $\begin{array}{c}\text { This work } \\
\text { diffuse } \\
\text { gamma } \\
\text { rays }\end{array}$ & $\begin{array}{c}\text { This work } \\
\text { galactic } \\
\text { gamma } \\
\text { rays }\end{array}$ & HR 2003 & $\begin{array}{c}\text { Fermi } \\
\text { HR 2004 } \\
\text { 2AT }\end{array}$ & $\begin{array}{c}\text { LHC } \\
\text { CMS } \\
\text { virtual } \\
\text { graviton }\end{array}$ \\
\hline $\mathrm{n}=3$ & 8.0 & 16 & 2.65 & 5.3 & 4.0 \\
$\mathrm{n}=4$ & 1.1 & 1.9 & 0.43 & 0.74 & 3.0 \\
\hline \hline
\end{tabular}

Table 1. Limits on $\mathrm{M}^{*}$ in $\mathrm{TeV}$, for this work and previous references in the text.

$\mathrm{SN}$ and $\mathrm{BH}$ contribute almost equally, since the lower number is compensated by a higher emissivity.

- $f_{k k}$ the fraction of energy released in the form of gravitons, which is the parameter of interest since $M^{*} \sim$ $f_{K K}^{-1 /(n+2)}$

- $f_{\text {trap }}$, the fraction of gravitons trapped by the compact remnant, the same being true for $\mathrm{NS}$ and $\mathrm{BH}$, taken as $1 / 3$ for $\mathrm{n}=3,4,5$ ((Hannestad and Raffelt 2003) , table II)

- $\tau_{\gamma}$, the gamma ray lifetime and $\tau$, the mean total lifetime, which is approximated as $\tau_{\gamma} / 4$ taking into account other decay channels (electron/positron and neutrino/antineutrino pairs, see eq. 44 in (Hannestad and Raffelt 2003), with $\tau_{\gamma}=\tau_{e} / 2=\tau_{\nu}$. For $n>3, \tau$ is relatively short $(<100 \mathrm{Myr})$. Over this time-scale, the production rate of gravitons, proportional to the $\mathrm{SN}$ rate, can be considered as approximately constant. Thus a steady state situation prevails.

Each second, an energy of $1.3 \times 10^{44}$ erg times $f_{K K}$ is injected into the galaxy in the form of gravitons by SN explosions. Most of them fly away and are lost from the galaxy since only a fraction $f_{\text {trap }} \approx 1 / 3$ remain trapped around NS and BH. The steady state energy in the form of gravitons confined permanently around neutron stars is $q \tau$ with $q=f_{K K} f_{\text {trap }} \times 6 \times 10^{53}$ ergs times the SN rate of 2 per century, or $4 \times 10^{44} \mathrm{erg} \mathrm{s}^{-1}$.

For $f_{K K}=1$, the gamma ray luminosity is $L_{\gamma}=$ $2 f_{\text {trap }} q \tau / \tau_{\gamma}$, since one graviton produces $\sim q / 6$, almost independently of the number $\mathrm{n}$ of ED i.e. $6.7 \times 10^{43} \mathrm{ergs}^{-1}$. This figure is to be compared to the residuals left by other processes (cosmic rays and possibly dark matter), say $5 \times 10^{37} \mathrm{ergs}^{-1}$, i.e. $10 \%$ of the emission observed by FermiLAT (Fermi-LAT Collaboration et al. 2012). Our final result is now: $f_{K K}<1.3 \times 10^{-6}$. This compares to the careful analysis of the Fermi-LAT group of the lack of gamma emission of selected individual neutron stars, which leads to $f_{K K}=8.7 \times 10^{-3}$ for $\mathrm{n}=3$ and $7.4 \times 10^{-4}$ for $\mathrm{n}=4$.

\section{CONCLUSION}

In the framework of the ADD extra-dimensional model for gravity at the $\mathrm{TeV}$ scale, we have revisited the constraints on the maximum fraction of energy emitted in the form of massive (Kaluza-Klein) gravitons by core collapse supernovae, coming from the extragalactic gamma-ray background and from residual galactic emission. From these constraints, we derive limits on the mass parameter $\mathrm{M}^{*}$ of the model for a number of extra dimensions of 3 and 4 . Table 1 summarizes the limits on $\mathrm{M}^{*}$ obtained in this work and in previous references mentioned in the text. 


\section{ACKNOWLEDGEMENTS}

We thank Elisabeth Vangioni for useful discussions.

\section{REFERENCES}

Buchmueller, O., and 14 colleagues 2012. European Physical Journal C 72, 2020.

Arkani-Hamed, N., Dimopoulos, S., Dvali, G. 1999. Physical Review D 59, 086004.

Randall, L., Sundrum, R. 1999. Physical Review Letters 83, 3370.

Cassé, M., Paul, J., Bertone, G., Sigl, G. 2004. Physical Review Letters 92, 111102.

Hannestad, S., Raffelt, G. G. 2001. Physical Review Letters 87, 051301.

Hannestad, S., Raffelt, G. G. 2002. Physical Review Letters 88, 071301.

Fermi-LAT Collaboration, and 122 colleagues 2012. Journal of Cosmology and Astro-Particle Physics 2012, 012.

Astashenkov, M. O., Kisselev, A. V. 2018. Physical Review D 98, 123009.

Ackermann, M., and 143 colleagues 2015. The Astrophysical Journal 799,86 .

Ackermann, M., and 128 colleagues 2016. Physical Review Letters 116, 151105.

MØller, K., Suliga, A. M., Tamborra, I., Denton, P. B. 2018. Journal of Cosmology and Astro-Particle Physics 2018, 066.

Priya, A., Lunardini, C. 2017. Journal of Cosmology and AstroParticle Physics 2017, 031.

ATLAS collaboration, Aaboud, M., Aad, G. et al. J. High Energ. Phys. (2018) 2018: 126.

CMS collaboration (2018) , A.M. Sirunyan et al. Phys. Rev. D 97, 092005.

CMS collaboration (2018), A.M. Sirunyan, A. M. et al. Eur. Phys. J. C 78 (2018) 789

Horiuchi, S., and 7 colleagues 2018. Monthly Notices of the Royal Astronomical Society 475, 1363.

Hannestad, S., Raffelt, G. G. 2003. Physical Review D 67, 125008.

Hannestad, S., Raffelt, G. G. 2004. Physical Review D 69, 029901.

Pardo, K., Fishbach, M., Holz, D. E., Spergel, D. N. 2018. Journal of Cosmology and Astro-Particle Physics 2018, 048. 